\title{
Thin-Film III-V Photodetectors Integrated on Silicon-on-Insulator Photonic ICs
}

\author{
Joost Brouckaert, Student Member, IEEE, Gunther Roelkens, Student Member, IEEE, \\ Dries Van Thourhout, Member, IEEE, and Roel Baets, Senior Member, IEEE
}

\begin{abstract}
We critically assess recent progress in the integration of near-infrared photodetectors onto nanophotonic siliconon-insulator (SOI) waveguide circuits. Integration of thin-film InGaAs photodetectors is studied in detail. This method consists of bonding unprocessed III-V dies onto the SOI substrate using an intermediate adhesive layer. Both benzocyclobutene and spin-on glass are studied and compared as bonding agents. After the removal of the III-V substrate, the thin-film detectors are fabricated using wafer-scale-compatible processes and lithographically aligned to the underlying SOI waveguides. The process is compatible with the fabrication of InP/InGaAsP laser diodes on SOI. A new design of an evanescently coupled metal-semiconductormetal detector is proposed, proving the ability to obtain compact and highly efficient integrated InGaAs photodetectors.
\end{abstract}

Index Terms-Heterogeneous integration, photonic integrated circuit (IC), silicon-on-insulator (SOI), thin-film photodetectors.

\section{INTRODUCTION}

$\mathbf{S}$ INCE the introduction of silicon photonics by Soref in the mid to late 1980s, silicon-on-insulator (SOI) is rapidly emerging as a versatile platform for a variety of integrated nanophotonic components [1], [2]. This trend is fueled by several factors and research developments [3]. The SOI material system possesses unique optical properties owing to the transparency of silicon and silica for wavelengths typically used for optical communications $(1.31-1.55 \mu \mathrm{m})$ and the large refractive index difference between silicon $(n=3.45)$ and silica $(n=1.45)$. This allows the creation of very compact and high-density waveguide circuits, including the so-called photonic wires with bend radii as small as $1 \mu \mathrm{m}$ [4] and photonic crystals. Moreover, these waveguide structures can be fabricated on a wafer scale using standard complimentary metal-oxide-semiconductor processing techniques, including deep ultraviolet lithography [5]. Keeping in mind the worldwide silicon fabrication infrastructure available, it is clear that this evolution could revolutionize the photonics industry.

The compactness, the ability to obtain very close spacing of the waveguides, and the wafer-scale processing are crucial for the integration of a multitude of photonic functions onto a

Manuscript received July 28, 2006; revised November 10, 2006. This work was supported in part by the Institute for the Promotion of Innovation through Science and Technology (IWT Flanders) under Project SBO epSOC, the IST Network of Excellence ePIXnet, and the Interuniversity Attraction Poles (IAP)Photon network. The work of G. Roelkens was supported by the Fund for Scientific Research (FWO)

The authors are with the Interuniversity Microelectronics Center, Department of Information Technology (INTEC), Ghent University, 9000 Ghent, Belgium (e-mail: joost.brouckaert@intec.ugent.be; gunther.roelkens@intec. ugent.be; dries.vanthourhout@intec.ugent.be; roel.baets@intec.ugent.be).

Digital Object Identifier 10.1109/JLT.2007.891172 single chip. This has the same advantage as the ultralarge-scale integration of electrical functionalities: increase of performance and reliability, the possibility of creating compact components with complex functionality, and cost reduction by making use of high-yield wafer-scale processes.

\section{Active/Passive Integration}

For some photonic functions like efficient light emission and light detection in the near-infrared region of the spectrum, silicon does not have the appropriate optical properties and other materials needed to be integrated. In this paper, we focus on the integration of photodetectors on SOI waveguide circuits. Detection of light at wavelengths typically used for optical communications $(1.31$ and $1.55 \mu \mathrm{m})$ is not possible in silicon, which is naturally transparent in this region. One possibility for overcoming this is the integration with germanium-containing materials. There are three main approaches that have been proposed to integrate germanium, each one based on a different type of active layer: $\mathrm{Si}_{1-x} \mathrm{Ge}_{\mathrm{x}} / \mathrm{Si}$ heterostructures, $\mathrm{SiGe}$ or $\mathrm{Ge}$ quantum dots on $\mathrm{Si}$ layers, and the use of pure $\mathrm{Ge}$.

The objective of growing multiple quantum wells of $\mathrm{SiGe} / \mathrm{Si}$ is to obtain strained $\mathrm{SiGe}$ layers with a reduced bandgap as compared to unstrained SiGe layers. However, the lattice mismatch between $\mathrm{Si}$ and SiGe limits the deposited thickness to a critical value before the onset of dislocation nucleation. For the large Ge concentrations needed to extend the absorption wavelength to $1.55 \mu \mathrm{m}$, the thickness of a single strained layer that can be grown without dislocations is limited to $10-20 \mathrm{~nm}$ [6], and this results in rather low effective absorption coefficients. This technology was used to fabricate SOI waveguideintegrated photodetectors at 1.3 and $1.55 \mu \mathrm{m}$, but rather low responsivities $(0.1 \mathrm{~A} / \mathrm{W})$ and long detectors (several $100 \mu \mathrm{m})$ were reported [6], [7].

A second approach relies on the Stranski-Krastanow growth of pure $\mathrm{Ge}$ on $\mathrm{Si}$. This growth regime leads to the formation of Ge-rich quantum dots. Waveguide-integrated photodetectors using a vertical stacking of self-assembled quantum dot layers were reported [8]; however, absorption coefficients at $1.55 \mu \mathrm{m}$ remain low, resulting in long devices (several millimeters) with low responsivities $(0.25 \mathrm{~mA} / \mathrm{W})$.

The third and more promising approach to obtain high absorption at 1.3 and $1.55 \mu \mathrm{m}$ is the use of strained pure Ge layers. Because of the large lattice mismatch of $4.2 \%$ between $\mathrm{Si}$ and $\mathrm{Ge}$, special growth strategies need to be applied. One of these strategies is to grow a thin low-temperature Ge buffer layer followed by a high-quality strained Ge layer [9]. A number 
of vertically illuminated Ge-on-Si detectors that employ this low-temperature Ge buffer layer method have been reported. Colace et al. obtained responsivities of $0.25 \mathrm{~A} / \mathrm{W}$ at $1.55 \mu \mathrm{m}$ [10], and by using a resonant cavity, Dosunmu et al. reported responsivities of $0.73 \mathrm{~A} / \mathrm{W}$ at $1.54 \mu \mathrm{m}$ [11]. However, as far as we know, there are no published results of Ge detectors integrated with SOI waveguides that operate at $1.55 \mu \mathrm{m}$.

A totally different route reported in the literature to obtain absorption at $1.5 \mu \mathrm{m}$ is to dope the Si waveguide with optically active erbium (Er) ions. Er-doped light-emitting diodes in $\mathrm{Si}$ operating at $1.5 \mu \mathrm{m}$ have been made with a quantum efficiency of up to $10^{-4}$ at room temperature [12], but the reverse process can also take place: an Er-doped Si p-n junction can generate a photocurrent when illuminated with $1.5-\mu \mathrm{m}$ light. However, due to the low absorption efficiency, these detectors have a very low responsivity and have to be several centimeters long [13].

We believe that heterogeneous integration of III-V compound semiconductors and, in particular, the approach based on bonding unprocessed III-V dies onto SOI waveguide wafers, as will be described in Section III, can lead to higher performances: InGaAs has an unchallenged position in low dark current, high speed, and high sensitivity integrated near-infrared photodetectors. On top of that, for the fabrication of SOI waveguide-integrated laser diodes [14], integration of the III-V material is the only viable solution, and detectors and sources could be fabricated in the same processing steps using the same wafer-scale III-V technologies.

\section{HeTERogeneous InTEGRATION}

The integration of III-V photodetectors with SOI waveguides can be carried out in different ways, such as epitaxial growth of III-V material, flip-chip integration, and adhesive bonding. It is important to notice that this choice will also very strongly influence the way light is coupled from the SOI waveguide to the detector.

A first possible approach is the heteroepitaxial growth of III-V materials directly onto the SOI substrate. Detectors can be fabricated and aligned to the silicon waveguides by making use of wafer-scale lithographical techniques. This approach has the advantage of allowing wafer-scale processing; however, due to large lattice constant mismatch (InP/Si 8.1\% mismatch) and differing thermal expansion constants of III-V compound semiconductors and $\mathrm{Si}$, the heteroepitaxial growth will lead to large strain and high misfit dislocation densities, thereby reducing the optical quality of the layers and the performance and reliability of the detector [15]. Much research effort is spent on different techniques to overcome these problems, such as epitaxial lateral overgrowth [16], the use of an intermediate buffer ceramic layer [17], and the use of GaAs buffer layers and quantum dot layers [18]. The optical coupling between waveguide and detector can be realized by using a butt-coupling or an evanescent-coupling scheme, but it has not been demonstrated.

A second approach is the integration of a processed detector or detector array using a flip-chip bonding technique. In the literature, this approach is often referred to as hybrid integration. One method to couple light from the waveguide to the detector is to make use of a grating etched in the SOI waveguide

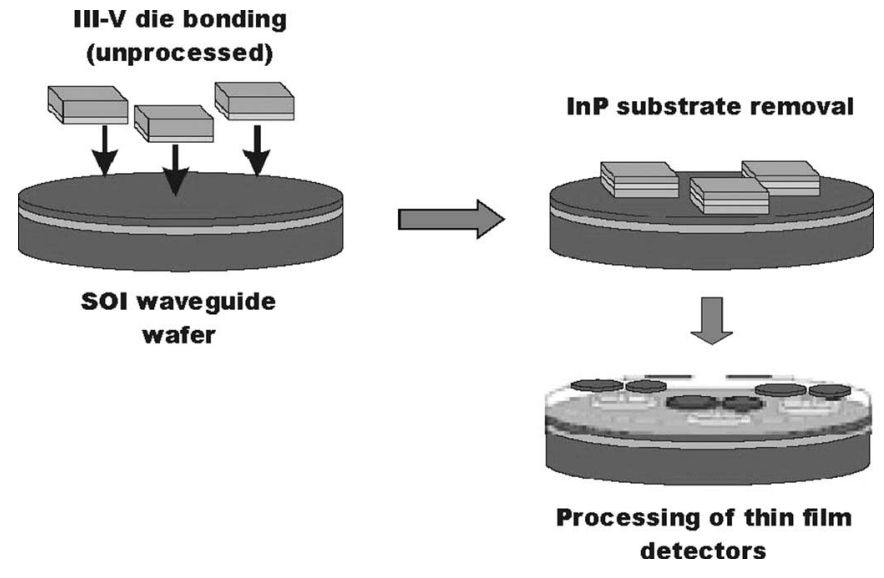

Fig. 1. Heterogeneous integration based on bonding of unprocessed III-V dies onto an SOI waveguide wafer. After die-to-wafer bonding, the InP substrate is removed, and the InGaAs detectors are processed, making use of waferscale lithographical techniques.

to couple light vertically out of the waveguide and into the detector on top of it. Another possibility is to butt couple the detector and waveguide [19]. Flip-chip integration has been used for many years and, as a result, is a relatively mature process. This method involves mating the surface of detectors with the SOI substrate by making use of solder bumps to connect the bottom contact of the detector with the contact pads on the SOI substrate. The top contact can be connected by a wire bond, or the detector can be designed so that both contacts are in the same plane. The main drawback of this approach is that integration is not carried out on a wafer-scale level and the alignment of the detector can be critical, resulting in a time-consuming and costly process. Using a larger detection area will decrease the necessary alignment accuracy but can increase the intrinsic capacitance of the device, which might be a limiting factor in high-speed high-bit-rate applications [20].

The proposed heterogeneous integration approach is described in Fig. 1. It consists of bonding unprocessed III-V dies (epitaxial layers down) onto a processed SOI substrate. Different bonding methods are possible and will be discussed in Section IV. This is the only nonwafer-scale process within the integration procedure, but because the dies are unprocessed, the alignment accuracy required for this step is limited, typically $>100 \mu \mathrm{m}$, so a rapid pick-and-place routine can be used. After bonding, the III-V semiconductor substrates are removed, obtaining defect-free epitaxial thin films bonded on an SOI waveguide wafer. Subsequently, the photodetectors are defined using wafer-scale processes and lithographically aligned to the underlying SOI waveguides. Different schemes to couple light from the SOI waveguide into a thin-film InGaAs detector will be discussed in Section V.

\section{DIE-TO-WAFER BONDING}

For the bonding of unprocessed III-V dies onto the SOI waveguide substrate, different bonding approaches are possible. As an optical transparent bonding layer is necessary, eutectic or metal bonding [21] can be excluded. The two main candidates for this application are direct wafer bonding with [22], [23] or without [24]-[26] an intermediate $\mathrm{SiO}_{2}$ layer 


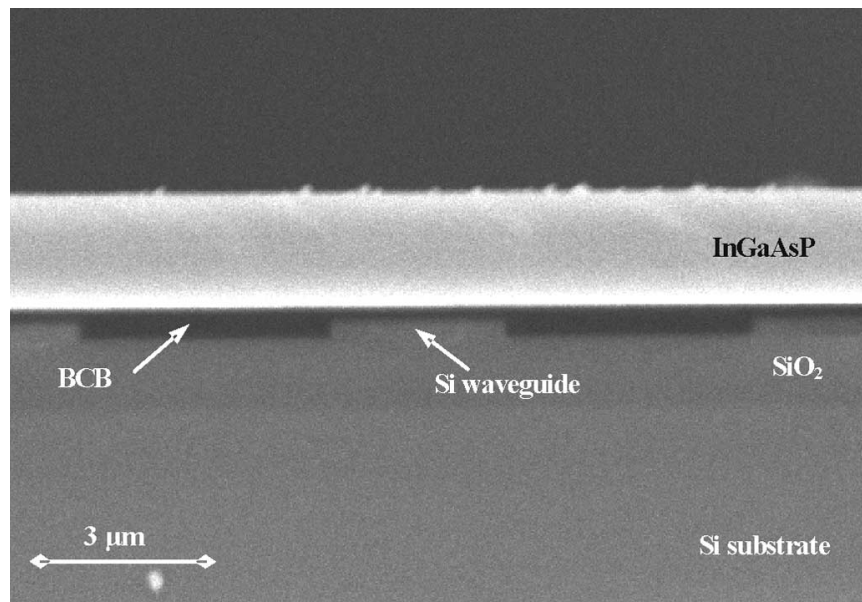

Fig. 2. BCB bonding.

and adhesive bonding [27], [28]. Highly efficient vertically incident InGaAs photodetectors on silicon have already been demonstrated using direct wafer bonding [26], [29], [30]. However, adhesive bonding offers a few advantages compared to direct bonding. Direct bonding often involves a high annealing temperature, which is undesirable for many applications. It imposes more severe requirements on flatness, microroughness, cleanliness, and chemistry of the two surfaces that have to be mated, imposing the need of advanced chemical mechanical polishing technology to obtain the appropriate surface conditions for bonding on processed wafers [22]. Using a polymer as bonding agent reduces these problems as the spin-coated polymer layer planarizes the wafer surface prior to bonding and can compensate inclusion of small particles between die and wafer. Therefore, we focused on adhesive bonding: both divinyldisiloxane benzocyclobutene (DVS-BCB) and spin-onglass (SOG) were investigated as an intermediate bonding layer. The waveguide structures are fabricated on $200-\mathrm{mm}$ SOI wafers with a silicon top layer of $220 \mathrm{~nm}$ and a buried oxide of $1 \mu \mathrm{m}$. The patterns were defined with 248-nm-deep UV lithography and transferred into the silicon using inductively coupled plasma-reactive ion etching. The fabrication process is described in detail in [5].

DVS-BCB is a heat-curable oligomer from Dow Chemicals. It can be spin coated in a large thickness range from about 1 to $25 \mu \mathrm{m}$ [31] and is highly transparent for wavelengths from 450 to $1550 \mathrm{~nm}$ [32]. The bonding process is well known and clearly described in the literature [28], [33]. To obtain efficient optical coupling between the SOI waveguide and the III-V photodetector on top of it, bonding layer thicknesses of less than $1 \mu \mathrm{m}$ are required, as will be discussed in Section V. Layer thicknesses down to $150 \mathrm{~nm}$ can be obtained by diluting the $\mathrm{BCB}$ with mesitylene [34]. A scanning electron microscope cross section of an InGaAsP epitaxial layer stack bonded on top of a structured SOI substrate is shown in Fig. 2. The InP substrate was removed by grinding down to about $60 \mu \mathrm{m}$ and finally chemically etched using $3 \mathrm{HCl}: 1 \mathrm{H}_{2} \mathrm{O}$ until an InGaAs etch stop layer is reached. The silicon waveguides are $3 \mu \mathrm{m}$ wide and $220 \mathrm{~nm}$ high, and the BCB layer on top of the waveguides is $200 \mathrm{~nm}$ thick.

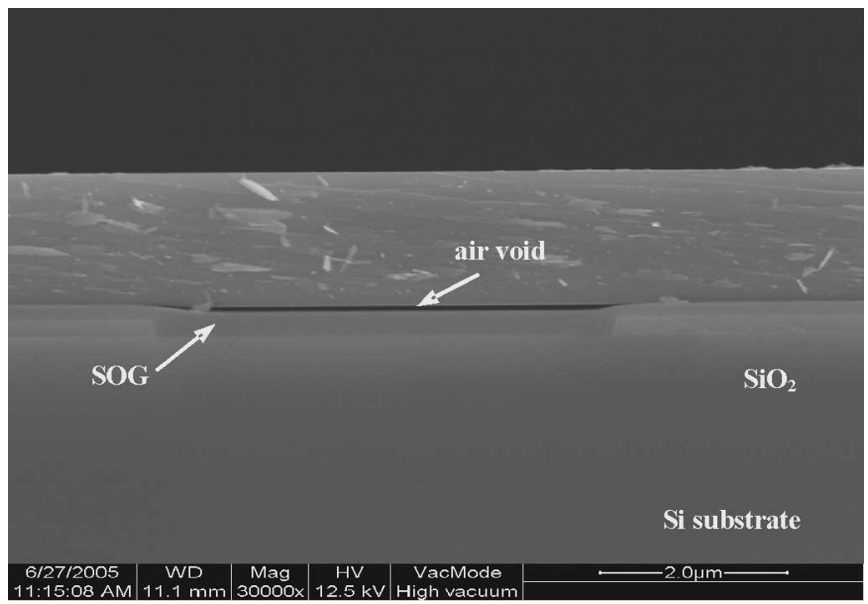

Fig. 3. SOG bonding.

SOG can also be used to obtain a thin bonding layer [35]. For the experiments carried out, we used Accuglass T-11 from Honeywell. This is a methylsiloxane type of SOG and can be spin coated in thicknesses ranging from 100 to $400 \mathrm{~nm}$. The bonding procedure started with sample cleaning. The InP wafer was cleaved into $0.5 \times 0.5 \mathrm{~cm}$ dies and then cleaned. These dies were bonded on both unprocessed Si samples and structured SOI samples. After spin coating the SOG on the $\mathrm{Si} / \mathrm{SOI}$ samples, they are placed on a hot plate at $150{ }^{\circ} \mathrm{C}$ for $5 \mathrm{~min}$ to remove solvents. Then, the InP dies are bonded at room temperature in a vacuum environment. To increase bonding strength, samples are cured in nitrogen ambient for $2 \mathrm{~h}$ at $300^{\circ} \mathrm{C}$. After substrate removal, an epitaxial III-V film bonded on a Si sample is obtained without any delamination or interfacial voids. However, bonding on structured SOI samples is less reproducible. As can be seen in Fig. 3, the bad planarizing properties of SOG give rise to air voids at the SOG/InP interface. These weak unbonded parts of the III-V film tend to crack or delaminate during substrate removal. Planarizing the SOI surface prior to bonding could solve these problems.

Although the topography of the SOI samples and the bonding layer thickness in both figures are equal, it is clear that BCB has better planarizing properties, and as a consequence of that, the $\mathrm{BCB}$ bonding process on structured substrates is more reproducible.

\section{Coupling Schemes}

In the following section, the different ways to couple light from the SOI waveguide into the detector are investigated. A usable coupling scheme needs to be compact and fabrication tolerant. On top of that, it should have an as large as possible spectral bandwidth and should make high-speed and high-efficiency optical detection possible. Polarization independence is not required as the nanophotonic waveguide circuits under study are designed for TE polarization only. A polarization diversity configuration can be implemented to obtain polarization-insensitive photonic integrated circuits [36]. It is essential to distinguish external quantum efficiency, which includes fiber coupling, from internal quantum efficiency, as defined in this paper, which indicates only the 


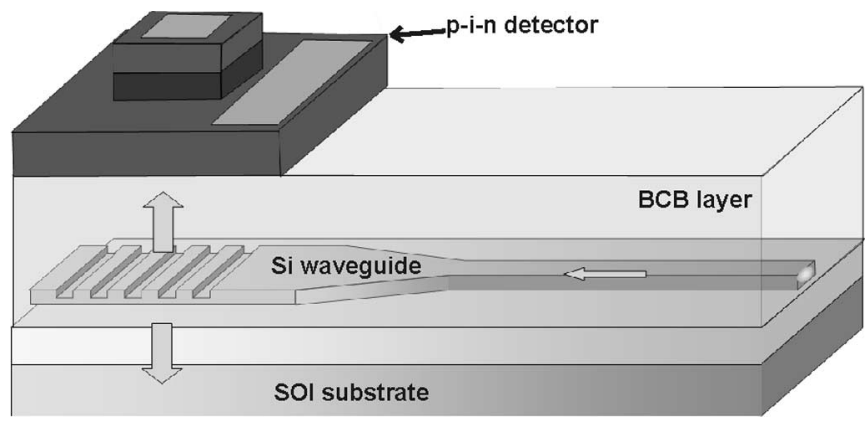

Fig. 4. Schematic view of an SOI waveguide-integrated p-i-n detector.

waveguide-to-detector coupling efficiency and is the relevant parameter for comparing different coupling schemes.

\section{A. Vertically Coupled Detector}

A straightforward way to couple light from a nanophotonic SOI waveguide into a detector bonded on top is to make use of a grating to diffract the light out of the SOI waveguide, through the bonding layer, into the detector. A schematic picture is shown in Fig. 4. In this approach, relatively thick bonding layers $(>1 \mu \mathrm{m})$ can be used, imposing more relaxed surface quality requirements as compared to the use of thin bonding layers.

We previously reported a vertically coupled $\mathrm{p}-\mathrm{i}-\mathrm{n}$ detector based on this approach [37]. The dark current of the $10 \times$ $10 \mu \mathrm{m}$ device is $0.3 \mathrm{nA}$ at a reverse bias of $1 \mathrm{~V}$, and the measured responsivity is $0.02 \mathrm{~A} / \mathrm{W}$ at $1.55 \mu \mathrm{m}$. This low value is due to the use of a nonoptimized p-i-n layer structure. Simulation results showed that efficient detection up to wavelengths of $1.6 \mu \mathrm{m}$ can be achieved with a quantum efficiency of $65 \%$ at $1.55 \mu \mathrm{m}$ using a p-i-n structure with a $2-\mu \mathrm{m}$-thick InGaAs absorption layer. The theoretical bandwidth is approximately $12 \mathrm{GHz}$ [38]. The maximal obtainable efficiency is however limited due to diffraction loss to the substrate.

For a given coupling efficiency from the SOI waveguide to the detector (including diffraction loss to the substrate), the quantum efficiency of the device will increase if a thicker InGaAs absorbing layer is used. To maintain high efficiency, relatively thick absorbing layers are needed. This implies a tradeoff between detector quantum efficiency and the transittime-limited bandwidth, which is inherent for most top- or bottom-illuminated detectors [38], [39]. This tradeoff can be circumvented by decoupling the dependence of efficiency on absorbing layer thickness using guided-wave detectors. This implies another way of optical coupling from the waveguide to the detector, as described in the next section.

\section{B. Evanescently Coupled Detector}

It is known that edge illumination allows high bandwidths without sacrificing efficiency or spectral bandwidth, because the optical absorption path length is independent of the carrier transit length [38]. The guided-wave detector-coupling scheme described in this section results in a device that is edge illuminated and thus offers the potential for efficient high-bandwidth operation.

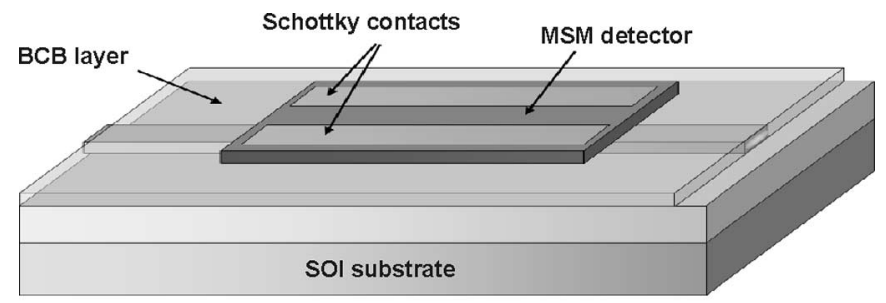

(a)

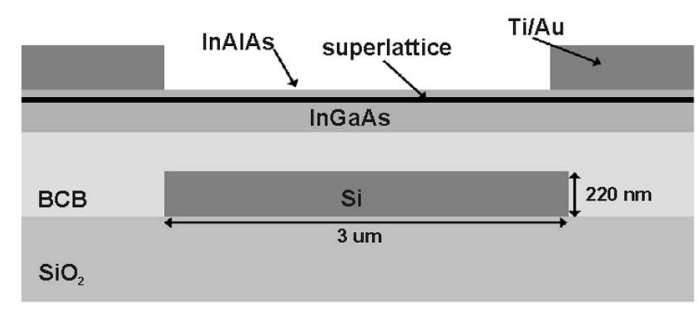

(b)

Fig. 5. (a) Three-dimensional and (b) cross-sectional views of waveguideintegrated MSM detector.

The proposed design is based on the principle of coupled mode theory. By using a thin bonding layer $(\sim 200 \mathrm{~nm})$ and by proper design to obtain phase matching between the optical mode in the SOI waveguide and the detector waveguide mode, the optical coupling between guide and detector will occur [40]. To obtain phase matching, the height of the detector layer stack will be close to the height of the silicon waveguides $(220 \mathrm{~nm})$ because the refractive indices of silicon and InGaAs are comparable in magnitude. This thin-layer stack can have a negative influence on efficiency: The top metal contact of a p-i-n detector will be in close proximity with the detector mode, thereby introducing excessive loss. Optical absorption in the metal contacts can be avoided by integrating a metal-semiconductor-metal (MSM) guided-wave detector. A schematic picture of the proposed design is shown in Fig. 5(a), while a cross-sectional view is shown in Fig. 5(b).

The material structure of the thin-film MSM detector consists of a 40-nm InAlAs Schottky barrier enhancement layer, a 20-nm InAlAs/InGaAs superlattice layer to decrease carrier trapping by the bandgap discontinuity between $\operatorname{In}_{0.52} \mathrm{Al}_{0.48} \mathrm{As}$ and $\mathrm{In}_{0.53} \mathrm{Ga}_{0.47} \mathrm{As}$, and a $145-\mathrm{nm} \mathrm{In}_{0.53} \mathrm{Ga}_{0.47}$ As absorption layer. All layers are not intentionally doped and lattice matched to the InP substrate. Two Schottky electrodes (Ti/Au, $20 \mathrm{~nm} /$ $200 \mathrm{~nm}$ ) are deposited on top, with the same spacing as the SOI waveguide, as can be seen in Fig. 5. An overview of the refractive indices of the materials used in the simulations is shown in Table I.

Lateral confinement of the detector waveguide mode is obtained by the two Ti/Au Schottky electrodes. The large imaginary part of the refractive indices of $\mathrm{Ti}$ (4.62) and $\mathrm{Au}$ (9.81) at $1.55 \mu \mathrm{m}$ lowers the effective refractive index of the fundamental slab mode of slice A as compared to slice B, and this causes lateral confinement, as can be seen in Fig. 6(b). As a consequence, the absorption loss in the Ti/Au electrodes is small, as will be calculated in this section, and on top of that, no waveguide ridge has to be defined, strongly simplifying the processing. An MSM detector offers, as compared to 
TABLE I

REFRACTIVE INDICES OF MATERIALS

\begin{tabular}{lccc}
\hline \hline \multicolumn{1}{c}{ Material } & Refractive index & $\begin{array}{c}\text { Absorption coeff. } \\
\left(\mathrm{cm}^{-1}\right)\end{array}$ & Ref. \\
\hline $\mathrm{Si}$ & 3.476 & - & \\
$\mathrm{SiO}_{2}$ & 1.444 & - & \\
$\mathrm{BCB}$ & 1.54 & - & \\
$\mathrm{In}_{0.53} \mathrm{Ga}_{0.47} \mathrm{As}$ & $3.595-0.096 \mathrm{j}$ & 7820 & 42 \\
$\mathrm{In}_{0.52} \mathrm{Al}_{0.48} \mathrm{As}$ & 3.202 & - & \\
$\mathrm{Ti}$ & $3.69-4.62 \mathrm{j}$ & 374560 & 44 \\
$\mathrm{Au}$ & $0.559-9.81 \mathrm{j}$ & 795330 & 45 \\
\hline \hline
\end{tabular}

Refractive indices of materials at $\lambda=1.55 \mu \mathrm{m}$ used in the simulations.

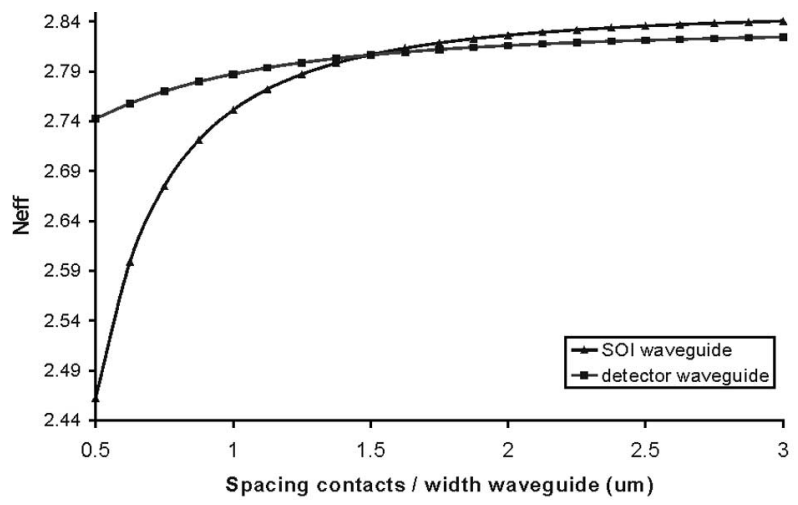

(a)
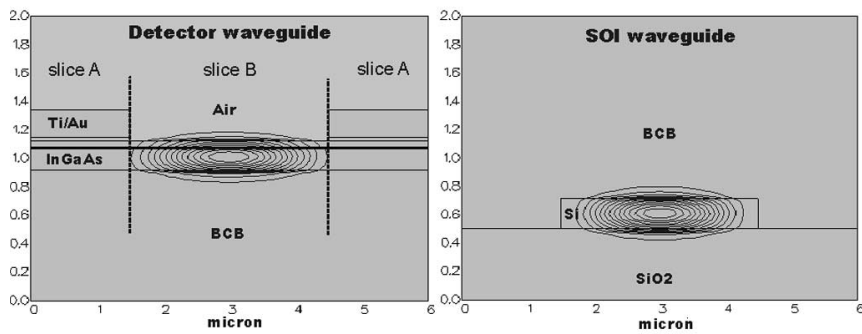

(b)

Fig. 6. (a) Real part of the effective refractive index of the TE ground modes of the decoupled detector waveguide and decoupled SOI waveguide as a function of contact spacing and waveguide width, respectively, and (b) corresponding mode profiles.

p-i-n-based devices, other important advantages: Its low capacitance per unit area makes it especially suitable for electrical circuits requiring a low input capacitance, it is easy to fabricate, and the epitaxial structure is free of highly doped layers [41].

Fig. 6(a) shows the (real part of the) effective refractive index (Neff) of the TE ground modes of the decoupled detector and SOI waveguide as function of Schottky contact spacing and waveguide width, respectively. The corresponding mode profiles are shown in Fig. 6(b). Using deep-UV lithography, contact spacings down to $200 \mathrm{~nm}$ are easily obtainable, but here, we limited ourselves to $1 \mu \mathrm{m}$, which is compatible with standard optical contact lithography. Phase mismatch of the decoupled waveguide modes smaller than $1.3 \%$ can be obtained with spacings/widths from 1 to $3 \mu \mathrm{m}$ using the layer structure described above. As absorption resonances occur when the (real) propagation constants of the fundamental modes of the decoupled waveguides coincide, the smaller is the phase mismatch, the higher is the detector absorption per unity of length.

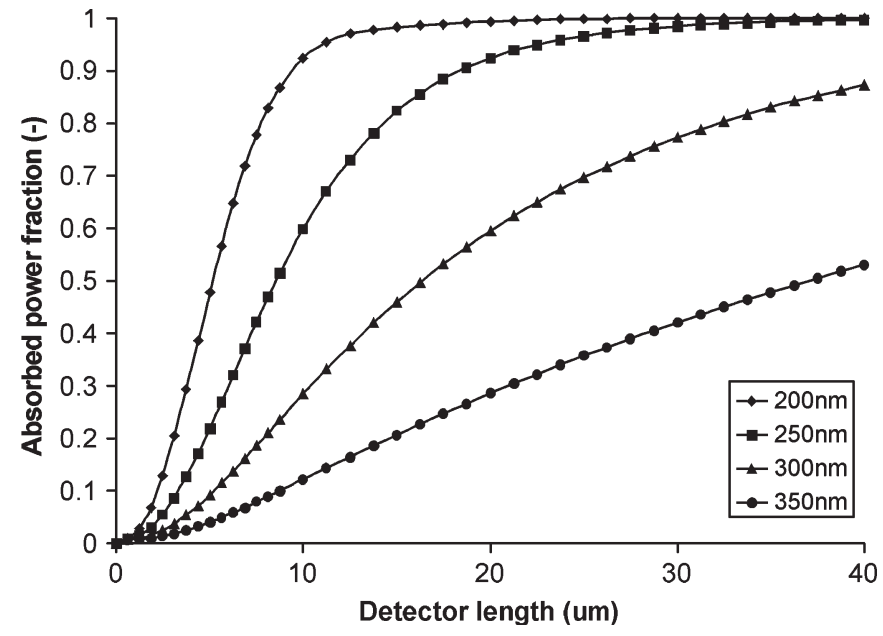

Fig. 7. Absorption as a function of detector length for different bonding layer thicknesses.

A fully vectorial 3-D simulation tool based on eigenmode expansion has been used to calculate the absorbed power as a function of detector length (Fig. 7). The simulated structure has a contact spacing and waveguide width of $3 \mu \mathrm{m}$. When having a 200-nm-thick BCB bonding layer between the SOI waveguide and the detector, $95 \%$ of the power is absorbed for detector lengths as short as $11 \mu \mathrm{m}$. It is well known from coupled mode theory that when the spacing between the two waveguides increases, the couple length will increase. As a consequence of that, the detector efficiency for a given length will decrease for increasing bonding layer thickness, as can be seen in Fig. 7. Bonding with $200 \mathrm{~nm}$ of BCB has been demonstrated as mentioned in Section IV.

To estimate the loss due to absorption in the Ti/Au Schottky contacts, we calculated the absorbed power as a function of detector length for both the real structure and the structure where we only take into account absorption in the Schottky contacts and no absorption in the InGaAs layers. However, this is an approximation because setting the imaginary part of the refractive index of InGaAs to zero slightly influences the field profile. The results are shown in Fig. 8. When we only consider absorption in the Schottky contacts, power in the structure is lost but at a very slow pace. From this approximated method, we can conclude that loss due to absorption in the Schottky contacts is limited (smaller than $10 \%$ of input power). This is caused by the fact that the contacts are used to obtain lateral waveguide confinement as explained earlier in this section.

In Fig. 9, the absorbed power as a function of wavelength is shown for detectors with lengths of 10,20 , and $50 \mu \mathrm{m}$ and with a $3-\mu \mathrm{m}$-wide detector and SOI waveguide. The dispersion relations of all the materials were taken into account in this simulation; however, the main contribution is due to the lower absorption coefficient of InGaAs at larger wavelengths [42], as can be seen in Fig. 9. There is a sharp drop in absorbed power at around $1.65 \mu \mathrm{m}$, which corresponds with the bandgap of InGaAs lattice matched to InP. By increasing the detector length, the spectral bandwidth can be improved; however, the detector capacitance and dark current will rise. The efficiency of the $10-\mu \mathrm{m}$-long detector increases for longer wavelengths 


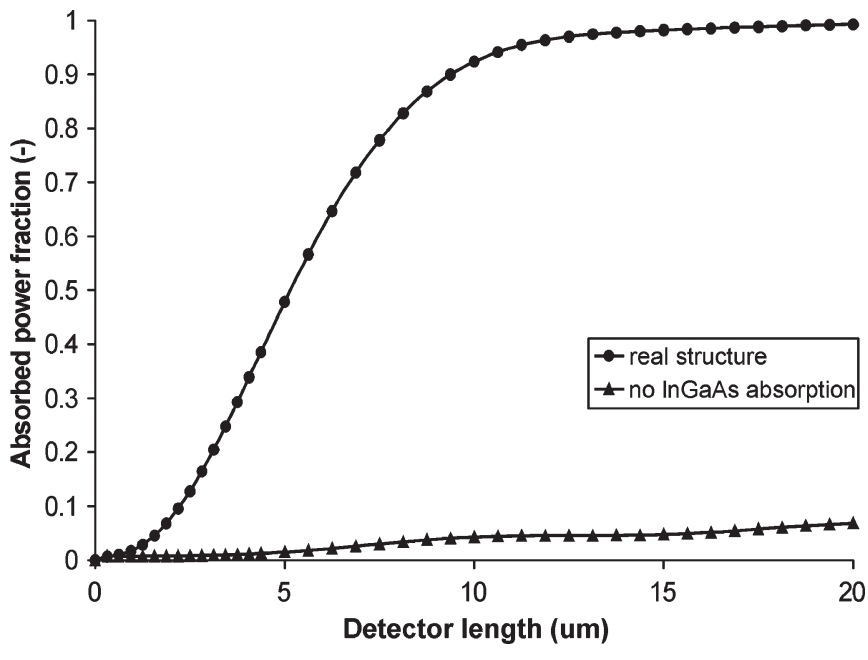

Fig. 8. Influence of optical absorption in the Schottky contacts (Ti/Au). One curve represents the real structure. The other only takes metal absorption into account and no absorption in the InGaAs layers.

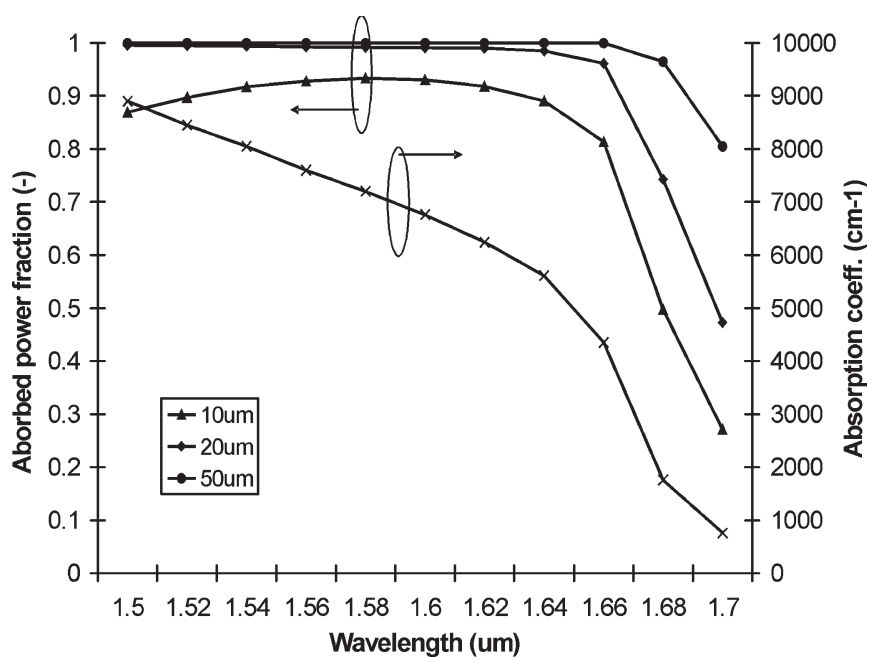

Fig. 9. Absorption coefficient of InGaAs and spectral bandwidth of the MSM detector; parameter is detector length.

before the sharp drop at $1.65 \mu \mathrm{m}$, as can be seen in Fig. 9. This can be explained by the fact that phase matching is more perfect at slightly higher wavelengths, and so, the effect of the lower absorption coefficient is cancelled.

The transit-time-limited bandwidth is mainly determined by the contact spacing, the thickness of the InGaAs layer $(145 \mathrm{~nm})$, and the spatial distribution of carriers in the absorption layer. The thin absorption layer has a positive influence on the transittime-limited bandwidth as the average carrier transit time is reduced. However, there will be some generation of carriers under the contact, where the electric field is weak, and this can deteriorate the response time of the device. For contact spacings of $1 \mu \mathrm{m}$, the bandwidth is limited by carrier transit time and is estimated to be approximately $15-20 \mathrm{GHz}$, as compared to literature examples [43].

\section{CONCLUSION}

This paper has reviewed different approaches to integrate near-infrared photodetectors onto passive SOI waveguide cir- cuits. A new design based on the adhesive bonding of unprocessed InP dies onto an SOI wafer was presented. Simulations of a waveguide-integrated InGaAs MSM detector were performed to predict its performance. It was shown that an internal quantum efficiency of more than $90 \%$ can be achieved for a 15- $\mu \mathrm{m}$-long device and a 200-nm intermediate BCB bonding layer. The device offers a high spectral bandwidth and a very simple processing. By tuning the detector length or bonding layer thickness, a fraction of the optical power can be detected for power monitor applications.

\section{REFERENCES}

[1] R. A. Soref and J. P. Lorenzo, "All-silicon active and passive guidedwave components for $\lambda=1.3$ and $1.6 \mu \mathrm{m}$," IEEE J. Quantum Electron., vol. QE-22, no. 6, pp. 873-879, Jun. 1986.

[2] M. Salib, L. Liao, R. Jones, M. Morse, A. Liu, D. Samara-Rubio, D. Alduino, and M. Paniccia. (2004, May). "Silicon photonics," Intel Technol. J., [Online]. 8(2), pp. 143-160. Available: http://developer. intel.com/technology/itj

[3] R. Baets, P. Dumon, W. Bogaerts, G. Roelkens, D. Taillaert, B. Luyssaert, G. Priem, G. Morthier, P. Bienstman, and D. Van Thourhout, "Siliconon-insulator based nano-photonics: Why, How, What for?" in Proc. 2nd IEEE Int. Conf. Group IV Photon. (IEEE Cat. No. 05EX1053), 2005, pp. $168-170$

[4] P. Dumon, G. Roelkens, W. Bogaerts, D. Van Thourhout, J. Wouters, S. Beckx, P. Jaenen, and R. Baets, "Basic photonic wire components in silicon-on-insulator," in Proc. 2nd IEEE Int. Conf. Group IV Photon. (IEEE Cat. No. 05EX1053), 2005, pp. 189-191.

[5] W. Bogaerts, R. Baets, P. Dumon, V. Wiaux, S. Beckx, D. Taillaert, B. Luyssaert, J. Van Campenhout, P. Bienstman, and D. Van Thourhout, "Nanophotonic waveguides in silicon-on-insulator fabricated with CMOS technology," J. Lightw. Technol., vol. 23, no. 1, pp. 401-412, Jan. 2005.

[6] H. Lafontaine, N. L. Rowell, S. Janz, and D. X. Xu, "Growth of undulating $\mathrm{Si} 0.5 \mathrm{Ge} 0.5$ layers for photodetectors at $\lambda=1.55 \mu \mathrm{m}$," J. Appl. Phys., vol. 86, no. 3, pp. 1287-1291, Aug. 1999.

[7] A. Splett, T. Zinke, K. Petermann, E. Kasper, H. Kibbel, H. J. Herzog, and H. Presting, "Integration of wave-guides and photodetectors in SiGe for $1.3 \mu \mathrm{m}$ operation," IEEE Photon. Technol. Lett., vol. 6, no. 1, pp. 59-61, Jan. 1994.

[8] M. El kurdi, P. Boucaud, S. Sauvage, G. Fishman, O. Kermarrec, Y. Campidelli, D. Bensahel, G. Saint-Girons, I. Sagnes, and G. Patriarche, "Silicon-on-insulator waveguide photodetector with $\mathrm{Ge} / \mathrm{Si}$ self-assembled islands," J. Appl. Phys., vol. 92, no. 4, pp. 1858-1861, Aug. 2002.

[9] J. M. Hartmann, A. Abbadie, A. M. Papon, P. Holliger, G. Rolland, T. Billon, J. M. Fedeli, M. Rouviere, L. Vivien, and S. Laval, "Reduced pressure-chemical vapor deposition of Ge thick layers on Si(001) for 1.3-1.55- $\mu$ m photodetection," J. Appl. Phys., vol. 95, no. 10, pp. 5905-5913, May 2004.

[10] L. Colace, G. Masini, G. Assanto, H. C. Luan, K. Wada, and L. C. Kimerling, "Efficient high-speed near-infrared Ge photodetectors integrated on Si substrates," Appl. Phys. Lett., vol. 76, no. 10, pp. 12311233, Mar. 2000.

[11] O. I. Dosunmu, D. D. Cannon, M. K. Emsley, L. C. Kimerling, and M. S. Unlu, "High-speed resonant cavity enhanced Ge photodetectors on reflecting Si substrates for 1550-nm operation," IEEE Photon. Technol. Lett., vol. 17, no. 1, pp. 175-177, Jan. 2005.

[12] S. Coffa, G. Franzo, and F. Priolo, "High efficiency and fast modulation of Er-doped light emitting Si diodes," Appl. Phys. Lett., vol. 69, no. 14, pp. 2077-2079, Sep. 1996.

[13] P. G. Kik, A. Polman, S. Libertino, and S. Coffa, "Design and performance of an erbium-doped silicon waveguide detector operating at $1.5 \mu \mathrm{m}$," J. Lightw. Technol., vol. 20, no. 5, pp. 834-839, May 2002.

[14] G. Roelkens, D. Van Thourhout, and R. Baets, "Coupling schemes for heterogeneous integration of III-V membrane devices and silicon-oninsulator waveguides," J. Lightw. Technol., vol. 23, no. 11, pp. 3827-3831, Nov. 2005.

[15] E. Peiner, A. Guttzeit, and H. H. Wehmann, "The effect of threading dislocations on optical absorption and electron scattering in strongly mismatched heteroepitaxial III-V compound semiconductors on silicon," J. Phys.-Condens. Matter, vol. 14, no. 48, pp. 13 195-13 201, Dec. 2002.

[16] Y. T. Sun, K. Baskar, and S. Lourdudoss, "Thermal strain in indium phosphide on silicon obtained by epitaxial lateral overgrowth," J. Appl. Phys., vol. 94, no. 4, pp. 2746-2748, Aug. 2003. 
[17] Z. Yu, R. Droopad, D. Jordan, J. Curless, Y. Liang, C. Overgaard, H. Li, A. Talin, T. Eschrich, B. Craigo, K. Eisenbeiser, R. Emrick, J. Finder, X. Hu, Y. Wei, J. Edwards, Jr., D. Convey, K. Moore, D. Marshall, J. Ramdani, L. Tisinger, W. Ooms, M. O'Steen, F. Towner, and T. Hierl, "GaAs-based heterostructures on silicon," in GaAs MANTECH Conf. Dig. Papers, 2002, pp. 276-279.

[18] Z. Mi, J. Yang, P. Bhattacharya, P. K. L. Chan, and K. P. Pipe, "High performance self-organized $\operatorname{In}(\mathrm{Ga})$ As quantum dot lasers monolithically grown on silicon," Proc. SPIE, vol. 6125, p. 612506, Mar. 2006.

[19] T. Mitze, M. Schnarrenberger, L. Zimmermann, J. Bruns, F. Fidorra, J. Kreissl, K. Janiak, S. Fidorra, H. Heidrich, and K. Petermann, "Hybrid integration of III/V lasers on a silicon-on-insulator (SOI) optical board," in Proc. 2nd IEEE Int. Conf. Group IV Photon. (IEEE Cat. No. O5EX1053), 2005, pp. 210-212.

[20] S. W. Seo, S. Y. Cho, S. Huang, J. J. Shin, N. M. Jokerst, A. S. Brown, and M. A. Brooke, "High-speed large-area inverted InGaAs thin-film metal-semiconductor-metal photodetectors," IEEE J. Sel. Topics Quantum Electron., vol. 10, no. 4, pp. 686-693, Jul./Aug. 2004.

[21] K. Hung-Fei, C. Sang-Yeon, J. Hall, and N. M. Jokerst, "InP/InGaAsP MQW thin film edge emitting lasers for embedded waveguide chip to chip optical interconnections," in Proc. IEEE LEOS Annu. Meeting Conf. (IEEE Cat. No.03CH37460), 2003, vol. 1, pp. 63-64.

[22] Q. Y. Tong and U. M. Gosele, "Wafer bonding and layer splitting for microsystems," Adv. Mater, vol. 11, no. 17, pp. 1409-1425, Dec. 1999.

[23] C. Monat, C. Seassal, X. Letartre, P. Viktorovitch, P. Regreny, M. Gendry, P. Rojo-Romeo, G. Hollinger, E. Jalaguier, S. Pocas, and B. Aspar, "InP 2D photonic crystal microlasers on silicon wafer: Room temperature operation at $1.55 \mu \mathrm{m}$," Electron. Lett., vol. 37, no. 12, pp. 764-766, Jun. 2001

[24] D. Pasquariello and K. Hjort, "Plasma-assisted InP-to-Si low temperature wafer bonding," IEEE J. Sel. Topics Quantum Electron., vol. 8, no. 1, pp. 118-131, Jan./Feb. 2002.

[25] T. Akatsu, A. Plossl, R. Scholz, H. Stenzel, and U. Gosele, "Wafer bonding of different III-V compound semiconductors by atomic hydrogen surface cleaning," J. Appl. Phys., vol. 90, no. 8, pp. 3856-3862, Oct. 2001.

[26] B. F. Levine, C. J. Pinzone, S. Hui, C. A. King, R. E. Leibenguth, D. R. Zolnowski, D. V. Lang, H. W. Krautter, and M. Geva, "Ultralowdark-current wafer-bonded Si/InGaAs photodetectors," Appl. Phys. Lett., vol. 75 , no. 14, pp. 2141-2143, Oct. 1999.

[27] V. Dragoi, T. Glinsner, G. Mittendorfer, B. Wieder, and P. Lindner, "Adhesive wafer bonding for MEMS applications," in Proc. SPIE-Int. Soc. Opt. Eng., 2003, vol. 5116, pp. 160-167.

[28] F. Niklaus, P. Enoksson, E. Kalvesten, and G. Stemme, "Low-temperature full wafer adhesive bonding," J. Micromech. Microeng., vol. 11, no. 2, pp. 100-107, Mar. 2001.

[29] A. R. Hawkins, W. Wu, P. Abraham, K. Streubel, J. E. Bowers, "High gain-bandwidth-product silicon heterointerface photodetector," Appl. Phys. Lett., vol. 70, no. 3, pp. 303-305, Jan. 1997.

[30] Y. Kang, P. Mages, A. R. Clawson, P. K. L. Yu, M. Bitter, Z. Pan, A. Pauchard, S. Hummel, and Y. H. Lo, "Fused InGaAs-Si avalanche photodiodes with low-noise performances," IEEE Photon. Technol. Lett., vol. 14, no. 11, pp. 1593-1595, Nov. 2002.

[31] Dow Chemicals, Processing Procedures For CYCLOTENE 3000 Series Resin. [Online]. Available: http://www.dow.com/cyclotene

[32] Y. G. Zhao, W. K. Lu, Y. Ma, S. S. Kim, S. T. Ho, and T. J. Marks, "Polymer waveguides useful over a very wide wavelength range from the ultraviolet to infrared," Appl. Phys. Lett., vol. 77, no. 19, pp. 2961-2963, Nov. 2000.

[33] Y. Ma, G. Chang, S. Park, L. W. Wang, and S. T. Ho, "InGaAsP thin-film microdisk resonators fabricated by polymer wafer bonding for wavelength add-drop filters," IEEE Photon. Technol. Lett., vol. 12, no. 11, pp. 14951497, Nov. 2000.

[34] G. Roelkens, D. Van Thourhout, and R. Baets, "Ultra-thin benzocyclobutene bonding of III-V dies onto SOI substrate," Electron. Lett., vol. 41, no. 9, pp. 561-562, Apr. 2005.

[35] D. Cengher, Z. Hatzopoulos, S. Gallis, G. Deligeorgis, E. Aperathitis, M. Androulidaki, M. Alexe, V. Dragoi, E. D. Kyriakis-Bitzaros, G. Halkias, and A. Georgakilas, "Fabrication of GaAs laser diodes on $\mathrm{Si}$ using low-temperature bonding of MBE-grown GaAs wafers with Si wafers," J. Cryst. Growth, vol. 251, no. 1-4, pp. 754-759, Apr. 2003.

[36] D. Taillaert, H. Chong, P. I. Borel, L. H. Frandsen, R. M. De La Rue, and R. Baets, "A compact two-dimensional grating coupler used as a polarization splitter," IEEE Photon. Technol. Lett., vol. 15, no. 9, pp. 1249-1251, Sep. 2003.
[37] G. Roelkens, J. Brouckaert, D. Taillaert, P. Dumon, W. Bogaerts, D. Van Thourhout, R. Baets, R. Notzel, and M. Smit, "Integration of InP/InGaAsP photodetectors onto silicon-on-insulator waveguide circuits," Opt. Express, vol. 13, no. 25, pp. 10 102-10 108, Dec. 2005.

[38] J. E. Bowers and C. A. Burrus, "Ultrawide-band long-wavelength p-i-n photodetectors," J. Lightw. Technol., vol. LT-5, no. 10, pp. 1339-1350, Oct. 1987.

[39] R. J. Deri, "Monolithic integration of optical wave-guide circuitry with III-V photodetectors for advanced lightwave receivers," J. Lightw. Technol., vol. 11, no. 8, pp. 1296-1313, Aug. 1993.

[40] M. R. Amersfoort, M. K. Smit, Y. S. Oei, I. Moerman, and P. Demeester, "Simple method for predicting absorption resonances of evanescentlycoupled waveguide photodetectors," in Proc. 6th Eur. Conf. Integr. Opt., Neuchitel, Switzerland, Apr. 18-22, 1993, pp. 2-40-2-41.

[41] J. B. D. Soole and H. Schumacher, "InGaAs metal-semiconductor-metal photodetectors for long wavelength optical communications," IEEE J. Ouantum Electron., vol. 27, no. 3, pp. 737-752, 1991

[42] E. Zielinski, H. Schweizer, K. Streubel, H. Eisele, and G. Weimann, "Excitonic transitions and exciton damping processes in InGaAs/InP," J. Appl. Phys., vol. 59, no. 6, pp. 2196-2204, Mar. 1986.

[43] W. A. Wohlmuth, P. Fay, K. Vaccaro, E. A. Martin, and I. Adesida, "Highspeed InGaAs metal-semiconductor-metal photodetectors with thin absorption layers," IEEE Photon. Technol. Lett., vol. 9, no. 5, pp. 654-656, May 1997.

[44] P. B. Johnson and R. W. Christy, "Optical-constants of transitionmetals- Ti, V, Cr, Mn, Fe, Co, Ni, and Pd," Phys. Rev. B, Condens. Matter, vol. 9, no. 12, pp. 5056-5070, Jun. 1974.

[45] E. D. Palik, Handbook of Optical Constants of Solids. New York: Academic, 1985. Academic Press Handbook Series.

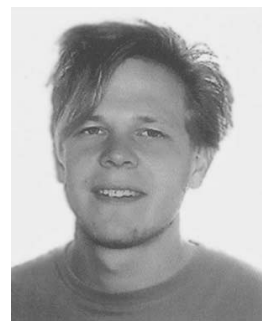

Joost Brouckaert (S'05) received the electronic engineering degree from Ghent University, Ghent, Belgium, in 2004.

Since 2004, he has been with the Department of Information Technology (INTEC), Ghent University. $\mathrm{He}$ is currently working in the field of heterogeneous integration and silicon-on-insulator nanophotonic components.

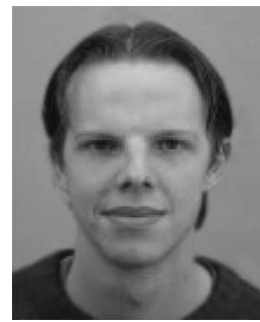

Gunther Roelkens (S'02) received the electronic engineering degree from Ghent University, Ghent, Belgium, in 2002

Since 2002, he has been with the Department of Information Technology (INTEC), Ghent University. $\mathrm{He}$ is currently working in the field of heterogeneous integration. 


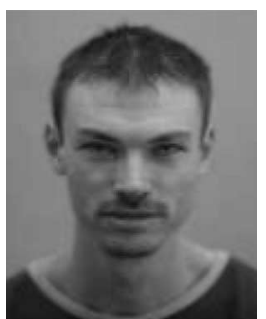

Dries Van Thourhout (S'95-M'95-S'99-M'00) received the physical engineering and Ph.D. degrees from Ghent University, Ghent, Belgium, in 1995 and 2000, respectively.

From October 2000 to September 2002, he was with Lucent Technologies, Bell Laboratories, Crawford Hill, NJ, working on the design, processing, and characterization of InPInGaAsP monolithically integrated devices. In October 2002, he joined the Department of Information Technology (INTEC), Ghent University, continuing his work on integrated optoelectronic devices. His main interests are heterogeneous integration by wafer bonding, intrachip optical interconnect, and wavelength-divisionmultiplexing devices.

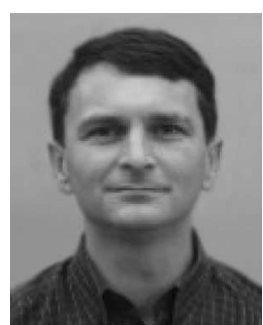

Roel Baets (M'88-SM'96) received the electrical engineering degree from Ghent University, Ghent, Belgium, in 1980, the M.Sc. degree in electrical engineering from Stanford University, Stanford, CA, in 1981, and the Ph.D. degree from Ghent University in 1984.

In 1981, he joined the Department of Information Technology (INTEC), Ghent University. Since 1989, he has been a Professor in the engineering faculty. From 1990 to 1994, he has also been a Part-Time Professor with the Technical University of Delft, Delft, The Netherlands. He has mainly worked in the field of photonic components. With about 300 publications and conference papers as well as about ten patents, he has made contributions to the design and fabrication of III-V semiconductor laser diodes, passive guided-wave devices, photonic integrated circuits, and microoptic components. He currently leads the Photonics Group, INTEC, which is an associated laboratory of the Interuniversity Microelectronics Center (IMEC), working on integrated photonic devices for optical communication, optical interconnect, and optical sensing.

Dr. Baets is a member of the Optical Society of America (OSA); the IEEE Lasers and Electro-Optics Society (LEOS), where he was the Chairman of the Benelux Chapter from 1999 to 2001 and is currently a member of the Board of Governors; the International Society for Optical Engineers (SPIE); and the Flemish Engineers Association. He has been a member of the program committees of the Optical Fiber Communications Conference (OFC), the European Conference on Optical Communication (ECOC), the IEEE Semiconductor Laser Conference, the European Solid-State Device Research Conference (ESSDERC), the Conference on Lasers and Electro-Optics (CLEO) -Europe, and the European Conference on Integrated Optics. 